\title{
Understanding phosphorus diffusion into silicon in a MOVPE environment for III-V on silicon solar cells
}

\author{
Elisa García-Tabarés , Diego Martín , Iván García , Ignacio Rey-Stolle
}

\begin{abstract}
A B S T R A C T
Dual-junction solar cells formed by a GaAsP or GalnP top cell and a silicon bottom cell seem to be attractive candidates to materialize the long sought-for integration of III-V materials on silicon for photovoltaic applications. When manufacturing a multi-junction solar cell on silicon, one of the first processes to be addressed is the development of the bottom subcell and, in particular, the formation of its emitter. In this study, we analyze, both experimentally and by simulations, the formation of the emitter as a result of phosphorus diffusion that takes place during the first stages of the epitaxial growth of the solar cell. Different conditions for the Metal-Organic Vapor Phase Epitaxy (MOVPE) process have been evaluated to understand the impact of each parameter, namely, temperature, phosphine partial pressure, time exposure and memory effects in the final diffusion profiles obtained. A model based on SSupremIV process simulator has been developed and validated against experimental profiles measured by ECV and SIMS to calculate P diffusion profiles in silicon formed in a MOVPE environment taking in consideration all these factors.
\end{abstract}

\section{Introduction}

Multijunction solar cell (MJSC) architectures offer a tremendous potential for achieving very high photovoltaic (PV) conversion efficiencies (up to $86 \%$ using an infinite number of junctions) [1]. State-of-the-art III-V MJSC designs are based on a substrate material, namely germanium, which is both costly and rare [2]. These factors have given rise to an active quest for alternative substrates, where silicon ( $\mathrm{Si}$ ) emerges as a natural choice as a result of its abundance and relative low cost. Interesting efforts in this direction were carried out in the past century and have reemerged strongly in recent years. One of the most successful approaches so far investigated is based on the use of a GaP nucleation layer to achieve a defect-free III-V template on Si [3-6]. On this template, GaAsP graded buffers can be grown onto which GaAsP or GalnP topcells of the adequate bandgap can be integrated, thus forming a GaAsP/Si [7-11] or a GaInP/Si [12] dual junction solar cell.

When manufacturing a multi-junction solar cell on silicon, one of the first processes to be addressed is the development of the bottom subcell, and particularly, the formation of the silicon subcell emitter. This paper reviews both experimentally and by simulations several key features for the optimization of the bottom subcell. Essentially, two alternatives exist for this process: (1) the emitter can be grown epitaxially, which implies that the III-V on $\mathrm{Si}$ cell will start with the homoepitaxial growth of n-type silicon on the p-type wafer; (2) mimicking what is done on III-V on Ge multijunction solar cell technology, the emitter of the silicon subcell can be formed by diffusion of a group- $V$ element at the initial stages of the heteroepitaxial process. The use of homoepitaxial growth has demonstrated to be a beneficial factor in the production of high quality GaP layers [4,8], though it introduces an additional degree of complexity in the epitaxial process. However, several groups have also reported high quality GaP layers without homoepitaxial silicon buffers $[3,9]$. Accordingly, we will focus on this strategy and consider the formation of the emitter from diffusion as is the case in conventional triple-junction solar cells based on germanium.

Phosphorus $(P)$ diffusion in crystalline silicon is a well-known phenomenon which has been thoroughly studied in the past 40 years [13]. However, the formation of the $n++$ emitter in the silicon subcell in a Metal-Organic Vapor Phase Epitaxy (MOVPE) environment is a complex process somewhat dissimilar to the traditional diffusion step in conventional photovoltaic (PV) technology. In this technology the emitter is formed by a process known as "phosphorous diffusion and gettering", thus phosphorous is supplied either from a phosphorous silicon glass (PSG) formed on the top of the wafer or by a spin-on source. In a MOVPE reactor, by the contrary, the emitter is formed by the diffusion of 
$\mathrm{P}$ atoms, resulting from the pyrolysis of a gaseous source, namely $\mathrm{PH}_{3}$, which will interact with the silicon surface. For the growth of III/V compounds on silicon in a MOVPE reactor, at the initial stage (pre-nucleation of GaP) several phosphorus compounds may coexist depending on the process followed and the temperature reached: atomic or molecular $P$ desorbed from the reactor walls and/or other heated parts (susceptor, exhaust, ...); $\mathrm{PH}_{3}$ intentionally injected and the byproducts of its pyrolysis $\left(\mathrm{PH}_{2}\right)$. All these compounds interact with the wafer surface to provide a final coverage of $P$ dimers and some displacement of silicon dimers (roughening). As a result of a certain phosphorus surface coverage at the temperatures used for the MOVPE process, diffusion of $\mathrm{P}$ into the wafer takes place. In the second stage in a process without a silicon homoepitaxial buffer, GaP nucleation starts but still an important phosphorus supply remains as a result of interdiffusion between the epilayer and the silicon substrate. Finally, in a third stage, during the growth of the graded buffer and the top subcell, the diffused phosphorus further penetrates into the substrate. In summary, the final junction depth will be the combined result of all these processes. In this paper, we will review-experimentally and using simulations based on SSupremIV-the formation of the emitter by the diffusion of $P$ into the silicon wafer, during the prenucleation stage, which mostly determines the emitter depth [14].

\section{Experimental}

The silicon substrates used in this work were p-type borondoped wafers oriented (100) with a miscut of $2^{\circ}$ towards the nearest (110) plane, grown by the Czochralski method, with a resistivity of $5-10 \Omega \mathrm{cm}$. Before loading wafers into the reactor they were chemically etched in $\mathrm{HF}: \mathrm{H}_{2} \mathrm{O}$ solution. They were dried afterwards with highly pure $(5 \mathrm{~N}) \mathrm{N}_{2}$. The experiments were carried out in a horizontal, research-scale MOVPE reactor (AIX200/4), equipped with an IR-lamp heater. All the quartz and graphite components in the reactor chamber were installed clean and heated to $850^{\circ} \mathrm{C}$ before each series of experiments. The carrier gas was Pd-purified $\mathrm{H}_{2}$, and the phosphorus precursor used was high purity phosphine $\left(\mathrm{PH}_{3}\right)$.

In order to assess the role of different variables on the formation of the emitter, two types of experiments were carried out: (1) exposure of wafers to a single annealing step under different phosphine partial pressures, and (2) a combined treatment consisting of an initial annealing under phosphine followed by a phosphorus drive-in under hydrogen atmosphere.

Temperatures from 800 to $875^{\circ} \mathrm{C}$ were used in the experiments for two different reasons: first, in order to obtain a deep enough emitter, a high temperature is required to guarantee the diffusion of the phosphorus into the silicon. Second, it has been widely reported the necessity of an initial wafer annealing (at a temperature higher than $800^{\circ} \mathrm{C}$ ) in order to favor the thermal desorption of the native silicon oxide and to promote the formation of double steps. Hence, an optimal surface, where a defect-free heteroepitaxial growth can be carried out, is obtained $[15,16]$.

A side effect of this annealing under phosphine is the degradation of the substrate surface morphology. It has been described that the exposure of $\mathrm{Si}(100)$ surfaces to $\mathrm{PH}_{3}$ may result in surface roughening due to $\mathrm{Si}$ hydration and subsequent dimer displacement $[17,18]$. Consequently, the optimum diffusion conditions for the formation of the bottom subcell emitter have to be attained without degrading the morphology of the substrate. The samples were characterized using Atomic Force Microscopy (AFM) to assess the impact of the MOVPE environment on the surface morphology degradation, which occurs during the formation of the emitter. AFM scans were taken using a Digital Instruments-Multimode IIIa microscope working in tapping mode and images were processed using a free software tool [19].

Phosphorus diffusion profiles have been measured by means of Electrochemical Capacitance-Voltage profiling (ECV) using $\mathrm{NH}_{4} \mathrm{~F}$ / $\mathrm{HF}$ as electrolyte [20]. Some of the wafers have also been selected for measuring Secondary Ion Mass Spectrometry (SIMS) using positive ions (whose low impact energy will provide a better depth resolution in the first nanometers of the sample) and negative ions (which provide a better resolution deeper into the sample).

\section{Results}

Table 1 summarizes the MOVPE treatment followed by each wafer. An estimation of the emitter depth, according to the ECV profiles, is also included. The main roughness parameters of the samples obtained from AFM characterization are summarized in Table 2.

ECV diffusion profiles measured for samples that have suffered a single annealing step (samples \#1 to \#4) are shown in Fig. 1. Time exposure to phosphine was constant in all the cases and equal to $60 \mathrm{~min}$. Temperature and phosphine partial pressure were different for each case, as shown in Table 1 . The emitter depth was estimated by an extrapolation from a fit of the ECV profiles using a complementary-error function for samples \#1 to \#4. These fits have been plotted in the figure as thin solid lines. The bottom gray band corresponds to the wafer nominal dopant concentration range, considering the uncertainty given by the manufacturer. It should be noted that the emitter depths estimated by the extrapolation of those fits have been obtained without considering either the uncertainty of ECV measurements (ECV profiling measures the electrically active fraction of dopants, and then it can

Table 1

Description of the experiments carried out on each sample. All experiments were conducted at a reactor pressure of 900 mbar using $\mathrm{H}_{2}$ as carrier gas. The emitter depth has been estimated from ECV and SIMS measurements.

\begin{tabular}{|c|c|c|c|c|c|c|c|}
\hline \multirow[t]{2}{*}{ Sample } & \multicolumn{3}{|c|}{ Step 1 (P-predeposition) } & \multicolumn{3}{|c|}{ Step 2 (drive-in) } & \multirow{2}{*}{$\begin{array}{l}\text { Emitter } \\
\text { depth } \\
\text { (nm) }\end{array}$} \\
\hline & $\begin{array}{l}\mathrm{PH}_{3} \\
\text { pressure } \\
\text { (mbar) }\end{array}$ & $\begin{array}{l}\text { Temp } \\
\left({ }^{\circ} \mathrm{C}\right)\end{array}$ & $\begin{array}{l}\text { Time } \\
(\mathbf{m i n})\end{array}$ & $\begin{array}{l}\mathrm{PH}_{3} \\
\text { pressure } \\
\text { (mbar) }\end{array}$ & $\begin{array}{l}\text { Temp } \\
\left({ }^{\circ} \mathrm{C}\right)\end{array}$ & $\begin{array}{l}\text { Time } \\
(\mathbf{m i n})\end{array}$ & \\
\hline \#1 & 32.1 & 875 & 60 & - & - & - & $260-274$ \\
\hline \#2 & 32.1 & 830 & & & & & $189-200$ \\
\hline \#3 & 32.1 & 800 & & & & & $173-183$ \\
\hline$\# 4$ & 0.7 & 830 & & & & & $93-99$ \\
\hline \#5 & 32.1 & 830 & 30 & 0 & 875 & 60 & $130-141$ \\
\hline$\# 6$ & & 830 & 30 & & 830 & & $103-112$ \\
\hline \#7 & & 875 & 10 & & 875 & & $80-87$ \\
\hline$\# 8$ & & 830 & 10 & & 875 & 90 & $110-121$ \\
\hline
\end{tabular}

Table 2

Summary of roughness parameters measured by AFM, for all samples shown in Table 1.

\begin{tabular}{llcc}
\hline Sample & RMS roughness $(\mathrm{nm})$ & Skewness & Kurtosis \\
\hline as-received Si wafer & 0.34 & 0.05 & 3.01 \\
\#1 & 1.92 & 3.56 & 24.87 \\
$\# 2$ & 2.23 & -0.06 & 3.03 \\
$\# 3$ & 1.17 & 0.21 & 2.98 \\
$\# 4$ & 0.33 & -0.76 & 3.95 \\
$\# 5$ & 0.29 & 0.00 & 3.01 \\
\#6 & 0.38 & -0.07 & 3.70 \\
\#7 & 0.29 & 0.10 & 3.13 \\
\#8 & 0.22 & 0.11 & 3.08 \\
\hline
\end{tabular}




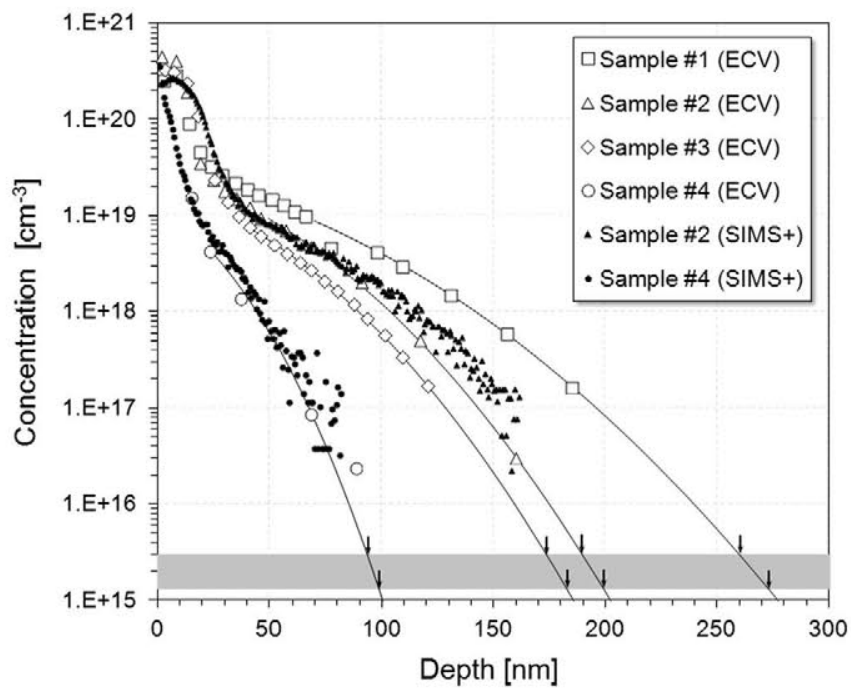

Fig. 1. ECV $P$ profiles of samples \#1 to \#4 (see Table 1). The thin solid lines represent fits of the tail of the ECV profiles based on the complementary error function used to estimate the emitter depth. SIMS profiles (positive ions) measured in samples \#2 and \#4 have been included for comparison. A range for the wafer nominal dopant concentration has been also included as a gray stripe on which the arrows indicate the limits for the estimated emitter depth in each case.

underestimate the total chemical concentration) or the uncertainty associated with the wafer doping (junction depth ranges are given based only on the uncertainty in the doping of the $\mathrm{Si}$ wafers). Thereby, both ECV and SIMS error margins are excluded, for the sake of simplicity, and thus emitter depths calculated are just an estimated value in order to be able to compare with theoretical values obtained from the simulations.

Fig. 1 compares the $P$ profiles obtained for samples \#1 to \#3, annealed under a partial pressure of $\mathrm{PH}_{3}$ of $32.1 \mathrm{mbar}$ for $60 \mathrm{~min}$ at 875,830 and $800^{\circ} \mathrm{C}$, respectively. P profiles obtained by SIMS (positive ions) and ECV correlate well and follow the typical kink and tail profile [13]. An initial shallow region (10-25 $\mathrm{nm}$ deep) with a very high doping is followed by a much deeper region showing a profile highly affected by the annealing temperature (emitter depths estimated for samples \#1 to \#3 range from 175 to $275 \mathrm{~nm})$. The depth of the initial saturated region $(-25 \mathrm{~nm})$ seems rather unaffected by temperature whilst the depth reached by the diffusion tail is strongly modulated by this parameter. According to Fig. 1, the saturation condition was reached for all samples \#1 to \#3 (i.e. for all temperatures in the range studied) measuring a surface $P$ concentration in the range of $3-5 \times 10^{20} \mathrm{~cm}^{-3}$, in agreement with values reported in the literature for the solid solubility of $\mathrm{P}$ in $\mathrm{Si}$ in this range of temperature $[21,22]$.

In order to assess the impact of the partial pressure of $\mathrm{PH}_{3}$, a new sample (\#4) was obtained by exposing the silicon wafer to $0.7 \mathrm{mbar}$ of $\mathrm{PH}_{3}$ at $830^{\circ} \mathrm{C}$ again for $60 \mathrm{~min}$. Fig. 1 also includes SIMS and ECV profiles for this sample (\#4), which show interesting differences with those of sample \#2, formed at the same temperature and time but under a $\mathrm{PH}_{3}$ partial pressure of 32.1 mbar. Despite the P surface concentration in both samples is similar, the depth of the saturated region in sample \#4 is roughly half of that of sample \#2 and the same occurs with the tail region of the profile, being in sample \#4 far less deep than in sample \#2 thereby producing a much shallower emitter. In other words, despite having a similar P-surface concentration at the same temperature $\left(830^{\circ} \mathrm{C}\right)$ for the same time $(60 \mathrm{~min})$, strong differences in $\mathrm{P}$ penetration are observed between both samples, as a result of differences in the gas phase in the MOVPE environment and consequently in the surface chemistry. As an indirect proof of the great differences in the surface processes occurring in both samples it is worth mentioning the large difference in RMS roughness measured, as shown in Table 2.

Since shallow emitters are preferable in our solar cell design, we explored the possibility of reducing the time exposure to phosphine (to $30 \mathrm{~min}$ ), maintaining a high phosphine partial pressure (32.1 mbar). In this case, we added an additional stepannealing under hydrogen-which is the so called $\mathrm{P}$ drive-in process intended to redistribute the phosphorus into the silicon. Fig. 2 shows the emitter profiles for samples \#5 to \#8, which have gone through the two step treatment (Table 1). In this case, as a result of the composite thermal treatment, the emitter depths were better estimated using a Gaussian function to fit the experimental profiles (thin solid lines in Fig. 2).

As shown in Fig. 2, the kink and tail profiles in these samples are severely modified. The surface concentration is greatly decreased (to values between $10^{20}$ and $10^{19} \mathrm{~cm}^{-3}$ ) and the saturated region is blurred. Of course, these two phenomena are the result of $\mathrm{P}$ in the surface region diffusing deeper into the substrate during the drive-in process. The effect of the temperature of the drive-in process can be assessed comparing samples \#5 (P drive-in at $\left.875^{\circ} \mathrm{C}\right)$ and $\# 6\left(\mathrm{P}\right.$ drive-in at $\left.830^{\circ} \mathrm{C}\right)$. In sample \#5 the surface concentration is lower and the tail penetrates deeper into the substrate as a result of a more intense in-diffusion due to the higher temperature. On the other hand, the effect of the parameters of the P-predeposition process (for the same drive-in duration and temperature) can be ascertained comparing the profiles of sample \#5 and \#7. In the case of sample \#7, the exposure to $\mathrm{PH}_{3}$ is just $10 \mathrm{~min}$ long at $875^{\circ} \mathrm{C}$ while conditions for sample \#5 are $30 \mathrm{~min}$ at $830^{\circ} \mathrm{C}$. In this case the short exposure in sample \#7 produces a shallower diffusion despite taking place at a higher temperature. This is in agreement with the results of sample \#1 and \#2 where the same difference in temperature didnot cause any difference in surface concentration. In this respect, we can conclude that, if sufficiently high partial pressures of phosphine are used, the duration of the $\mathrm{PH}_{3}$ exposure determines the depth into the wafer of the saturated region (i.e. in a first-order approximation the amount of the P loaded into the wafer) while the conditions of the drive-in determine the intensity of the redistribution (i.e. the emitter depth). Finally, sample \#8

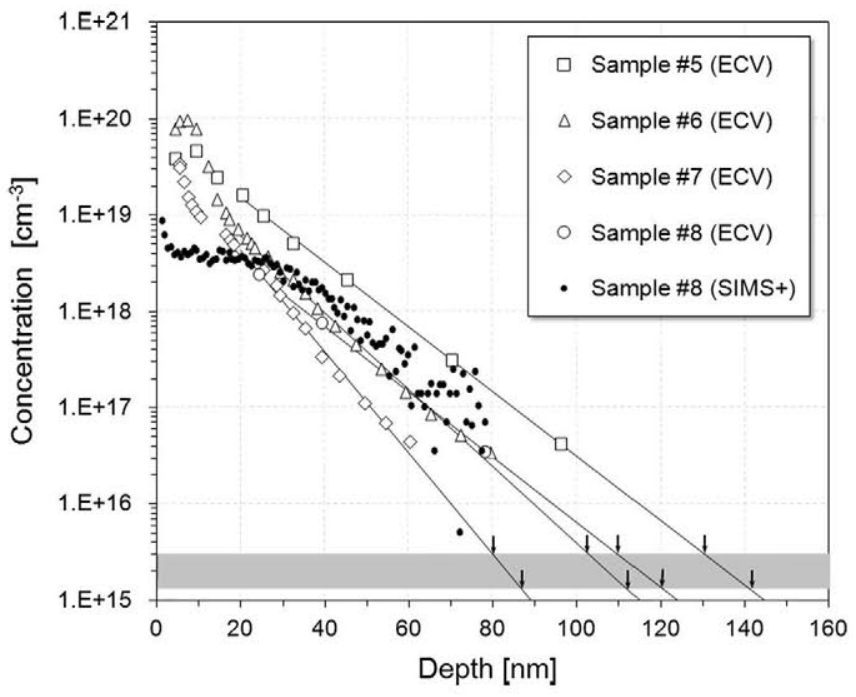

Fig. 2. ECV $P$ profiles of samples \#5 to \#8 (see Table 1). The thin dashed lines represent fits of the tail of ECV profiles based on a Gaussian function used to estimate the emitter depth. The SIMS profile (positive ions) measured in sample \#8 has been included for comparison. A range for the wafer nominal dopant concentration has been also included as a gray stripe on which the arrows indicate the limits for the estimated emitter depth in each case. 
exemplifies the effects of a long drive-in $(90 \mathrm{~min})$ where the profile has evolved into a pure tail with no kink.

The emitters formed in these experiments are shallower in all samples (between $80 \mathrm{~nm}$ and $140 \mathrm{~nm}$ ). In terms of emitter depth, these profiles are very similar to the ones obtained for the low phosphine partial pressure in Fig. 1 (sample \#4). Therefore, exposing the wafers to a high phosphine concentration during a short time will be enough to obtain a shallow emitter if an additional drive in step is carried out. Furthermore, the beneficial effect of the second step (hydrogen annealing) on the surface morphology becomes evident when comparing the RMS roughness (Table 2), since an important reduction of this value has been observed in the second group of samples, even when working with high phosphine partial pressures. Moreover, these RMS roughness values are very similar to those of as-received wafers, this being an indication of the total recovery of the surface morphology after the annealing in hydrogen, as reported by other authors [18,23].

\section{Discussion}

With the aim of having a better understanding of the diffusion profiles obtained, simulations of the phosphorus diffusions in $\mathrm{p}$ doped Si have been carried out and compared to the experimental data. SIMS measurements instead of ECV will be used for the comparisons, because of the fact that they represent the total dopant concentration, not only the electrically active fraction.

If the phosphorus surface concentration reaches saturation conditions in silicon, it is accepted that it diffuses through a two-stream mechanism: a vacancy-mediated process at high concentrations and an interstitially driven diffusion at lower $\mathrm{P}$ concentrations [24]. The superposition of these two fluxes results in the typical kink-and-tail profiles such as some of the ones shown in Fig. 1. To be able to theoretically reproduce such complex concentration profiles, we have chosen to simulate phosphorus diffusion profiles using the SSupremIV process simulator included in the Silvaco TCAD ATHENA( $\&$ software package [25]. The phosphorus doping profiles are calculated using a fully coupled diffusion model, in which the dopant and the non-equilibrium point defect populations-namely, lattice vacancies and silicon selfinterstitials-are directly represented and evolved in time. Therefore, three different sets of diffusion equations are used: one for phosphorus atoms, one for self-interstitials and one for vacancies. The phosphorus diffusivity values included in SSupremIV are dopant-concentration dependent by using the model proposed by Fair [26]:

$D(T, n)=D^{x}+D^{-}\left(\frac{n}{n_{i}}\right)+D^{=}\left(\frac{n}{n_{i}}\right)^{2} \quad$ where $D^{i}=D_{0}^{i} \exp \left(\frac{-E_{A}}{k T}\right)$

Vacancies and interstitials have different diffusivities than dopants. The values used in our simulations are those published in [27]. Each diffusivity coefficient is temperature-dependent following an Arrhenius-like dependence. Table 3 lists the

Table 3

Pre-exponential factor and activation energy for the non-zero Arrhenius-like diffusivity coefficients of phosphorus $\left(D^{x}, D^{-}, D^{=}\right)$ [26] and of vacancies and self-interstitials in silicon [27].

\begin{tabular}{lcc}
\hline III.a & $D_{0}\left(\mathrm{~cm}^{2} / \mathrm{s}\right)$ & $E_{A}(\mathrm{eV})$ \\
\hline$D^{x}$ & 3.85 & 3.66 \\
$D^{-}$ & 4.44 & 4.00 \\
$D^{=}$ & 44.20 & 4.37 \\
Interstitials & 600.00 & 2.44 \\
Vacancies & 0.10 & 2.00 \\
\hline
\end{tabular}

pre-exponential factors and activation energies used in the diffusion simulations.

From a physical viewpoint, the fully coupled diffusion model relies on the dilute approximation (i.e. the assumption that the concentration of dopant-defect pairs is much smaller than both the dopant and the defect concentrations). There is no subdivision of defects and dopant into paired and non-paired fractions. Then, the dopant-defect coupling is managed by introducing two empirical parameters, $f_{P V}$ and $f_{P I}$. The first parameter $f_{P V}$ is the fractional vacancy component of diffusion, and it accounts for the fraction of phosphorus atoms that diffuse via lattice vacancy defects under non-equilibrium conditions. Similarly, its complementary parameter $f_{P l}$, accounts for the fraction diffusing through self-interstitial defects in the same conditions. Both parameters are used in the flux equation for the phosphorous atoms, which diffusion is then highly influenced by the diffusion of vacancies and interstitials. In addition, in the fully coupled diffusion model the phosphorus flux term is also present in the continuity equations for the point-defect populations, allowing the diffusion of vacancies and interstitials to be also influenced by the diffusion of the dopant atoms. It is worth noting that $f_{P I}+f_{P V}=1$. However, it is already well-known [24] that the interstitially-driven mechanism clearly dominates when phosphorus diffuses into silicon, forcing $f_{P I}$ to be close to 1 . Then, according to the data found in [24], $f_{P V}$ has been fixed to 0.01 in all our diffusion simulations.

Point-defect recombination at interfaces and in the bulk is also modeled in $A T H E N A(\mathbb{B}$. It also allows modifying the process boundary conditions, as the injection of extra point defects from the surface into the substrate, which will be of special interest to account for $\mathrm{PH}_{3}-\mathrm{Si}$ reactions occurring at the silicon surface. Second-order effects as the capture or emission of interstitials by traps, dopant electrical deactivation and clustering, etc. are also modeled. More detailed explanations of the ATHENA( $)$ platform can be found in [25].

Fig. 3a shows the SIMS phosphorus profiles using positive and negative ions for sample \#2, which was exposed to a relatively high partial pressure of $32.1 \mathrm{mbar}$ of $\mathrm{PH}_{3}$ at $830^{\circ} \mathrm{C}$ during $60 \mathrm{~min}$. The use of positive and negative ions allows obtaining maximum resolution in the near surface region (positive ions) as well as in the sample bulk (negative ions). The leftmost solid line-labeled $0 \mathrm{~cm}^{-2} \mathrm{~s}^{-1}$-corresponds to the simulated diffusion profile for the nominal values of time and temperature and no vacancy injection. A surface concentration of $4 \times 10^{20} \mathrm{~cm}^{-3}$ has been set, matching the surface value determined by SIMS measurements. It can be clearly seen how poor the fitting to the experimental data is.

However, it is agreed that enhanced dopant diffusion in silicon is generally due to the generation of an extra concentration of point defects, particularly of vacancies in the saturated regions. In this sense, we have simulated the effect of having and extra population of vacancies at the silicon surface. Fig. 3a shows these new simulations as solid lines labeled with the extra generation rate for vacancies assumed in each case. If an extra flux of vacancies at the surface of around $10^{11} \mathrm{~cm}^{-2} \mathrm{~s}^{-1}$ is considered then the high-concentration part of the profile can be fitted quite accurately (red solid line in Fig. 3a). However, a clear underestimation of the profile in the tail region is still present. Many reports have shown that the diffusion rate in the tail region is highly correlated with the concentration of self-interstitials in the bulk [24]. This rise in self-interstitial concentration in the bulk is generally correlated with an increase in the generation of defects at the surface or with a decrease in their annihilation rate. Accordingly, Fig. 3b shows new calculations where the extra generation rate of vacancies at the surface has been fixed to $7 \times 10^{10} \mathrm{~cm}^{-2} \mathrm{~s}^{-1}$, which produced the best fit to the SIMS profile with positive ions in the saturated region, while different values for the self-interstitial generation rate at the surface have been 
a

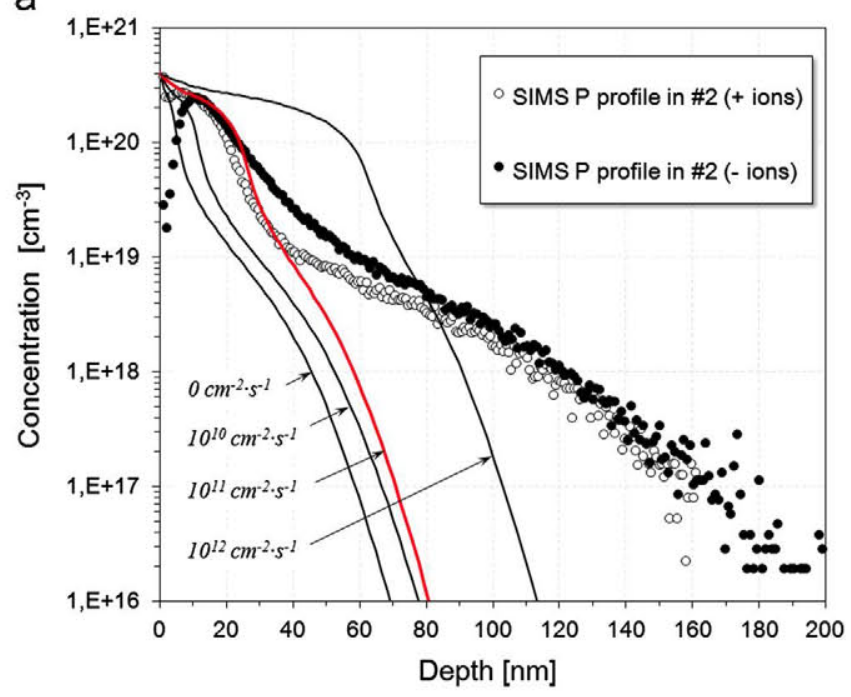

b

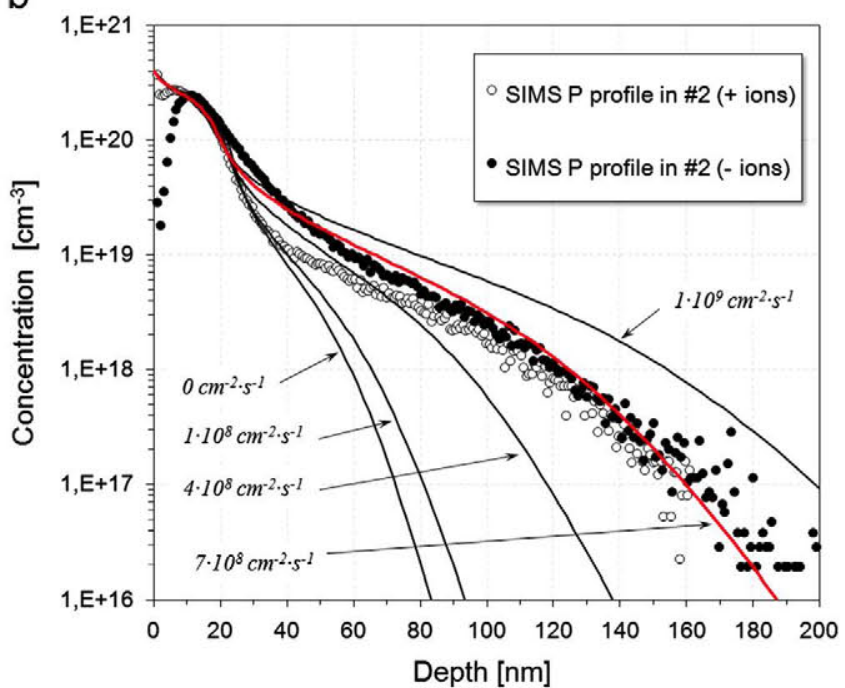

Fig. 3. (a) Comparison of the phosphorus SIMS profiles measured for sample \#2 and the simulation (thin solid lines), for different vacancy injection values (in $\mathrm{cm}^{-2} \mathrm{~s}^{-1}$ ). No interstitial injection has been included. (b) Same for a fixed vacancy injection of $7 \times 10^{10} \mathrm{~cm}^{-2} \mathrm{~s}^{-1}$ and as a function of the silicon self-interstitial injection (also in $\mathrm{cm}^{-2} \mathrm{~s}^{-1}$ ). (For interpretation of the references to color in this figure, the reader is referred to the web version of this article.)

assumed. These new simulations are plotted as solid lines in Fig. $3 \mathrm{~b}$ the label indicating the interstitial generation rate. For sample \#2, adding an extra generation rate of silicon selfinterstitials at the silicon surface of about $7 \times 10^{8} \mathrm{~cm}^{-2} \mathrm{~s}^{-1}$ (red solid line in Fig. $3 \mathrm{~b}$ ) increases sufficiently the bulk $\mathrm{Si}$ interstitial population without affecting the vacancy-mediated high-concentration part of the profile, and leads to a simulated phosphorus profile fairly close to the data measured by SIMS.

In summary, to obtain a reasonable fit to the experimental $P$ profiles as measured by SIMS we need to consider an important injection of vacancies $\left(7 \times 10^{10} \mathrm{~cm}^{-2} \mathrm{~s}^{-1}\right)$ with a much smaller simultaneous injection of self-interstitials $\left(7 \times 10^{8} \mathrm{~cm}^{-2} \mathrm{~s}^{-1}\right)$. It is well-known that the chemical interaction of silicon from the wafer with foreign species to form deposited films can be a source of point defects. For instance, the nitridation of a silicon wafer to form a layer of silicon nitride has been reported to represent an extrinsic source of vacancies, while silicon oxidation injects extra self-interstitials at the surface [24]. Obviously, in our case neither nitridation nor oxidation is present, but significant chemical interaction is known to occur between $\mathrm{PH}_{3}$ (and its byproducts) and the silicon wafer at our process temperatures. Moreover, in our case, the AFM measurements presented in the previous section have already shown how phosphine partial pressure affects the morphology of the silicon surface. The samples exposed to higher $\mathrm{PH}_{3}$ partial pressures at higher temperatures for longer times present a substantial increase in surface RMS roughness (shown in Table 2, which summarizes roughness data for all samples ) as a result of the proliferation of pits (holes), which were not present in the AFM scans of as-received wafers. In addition, surface skewness and surface kurtosis-which should be zero and three, respectively, for a flat morphology-deviate from their standard values. Larger deviations are observed in samples \#1 and \#3 indicating that these samples are not formed by sets of evenly distributed peaks and valleys of homogeneous heights [28]. All these morphological features are the result of $\mathrm{Si}$ hydration and subsequent dimer displacement. We speculate that the phenomenology behind this process is as follows: when large quantities of $\mathrm{PH}_{3}$ are present in the reactor for sufficient time, the wafer surface is etched significantly and a number of silicon atoms are removed from the pre-existing surface reconstruction; silicon atoms in the bulk diffuse towards the surface in a trend to restore thermodynamic equilibrium (i.e. minimizing surface energy by replenishing $\mathrm{Si}$ atoms for the surface reconstruction) creating vacancies mostly in the subsurface region. In such process, most of the displaced silicon would form silane (or other ionized hydride) and desorb into the gas phase. However, a minimum amount of the silicon/hydrogen complexes-1 out of each 100 atoms according to our calculations-could dissociate while being still adsorbed at the surface giving the chance to the silicon atom to diffuse back into the bulk, thus feeding the proposed extra flux of self-interstitials. The enhanced presence of vacancies and selfinterstitials in these samples is currently under investigation using Rutherford Backscattering (RBS) techniques. In particular, ion channeling experiments, which can provide very precise information on the lattice location of an impurity atom or defect in the $\mathrm{Si}$ lattice, are underway to further substantiate our hypothesis.

If this correlation between morphology degradation and point defect injection were correct, those samples with lighter exposure to $\mathrm{PH}_{3}$ (i.e. exposed to low partial pressures of phosphine or to high partial pressures for short times) and then better surface RMS roughness due to the lack of silicon displacement, should present less or even no enhancement in the diffusivity of the tail region. Fig. 4 presents the SIMS-measured phosphorus profile for sample \#4, exposed to $\mathrm{PH}_{3}$ for the same time and temperature than sample \#2 but with a significant reduction in the $\mathrm{PH}_{3}$ partial pressure from 32.1 to 0.7 mbar. In this case, the RMS surface roughness measured on sample \#4 is of $0.33 \mathrm{~nm}$, which is about the same roughness value measured on as-received wafers, indicating that this light $\mathrm{PH}_{3}$ exposure has had little effect (if any) on the wafer surface (see Table 2).

Comparing the simulations of the phosphorus profile to the SIMS data in Fig. 4, two main conclusions can be drawn. The first one is that, even having nearly the same P surface concentration of $4 \times 10^{20} \mathrm{~cm}^{-3}$, there is no clear evidence of dopant saturation in the first nanometers of the diffusion profile. This is consistent with having about 45 times less $\mathrm{PH}_{3}$ partial pressure, and then a lower surface coverage of $\mathrm{P}$ dimers of the surface. The second conclusion is that if, to simulate the profiles, we assume the same generation rates for vacancies and self-interstitials used in Fig. 3 for sample $\# 2$, then a clear overestimation of the diffusion profile is obtained (see black solid line in Fig. 4). On the contrary, if no injection of vacancies and self-interstitials is considered, the simulated $\mathrm{P}$ profile fits the measured data reasonably well (red solid line in Fig. 4). This suggests that without morphology degradation (and 


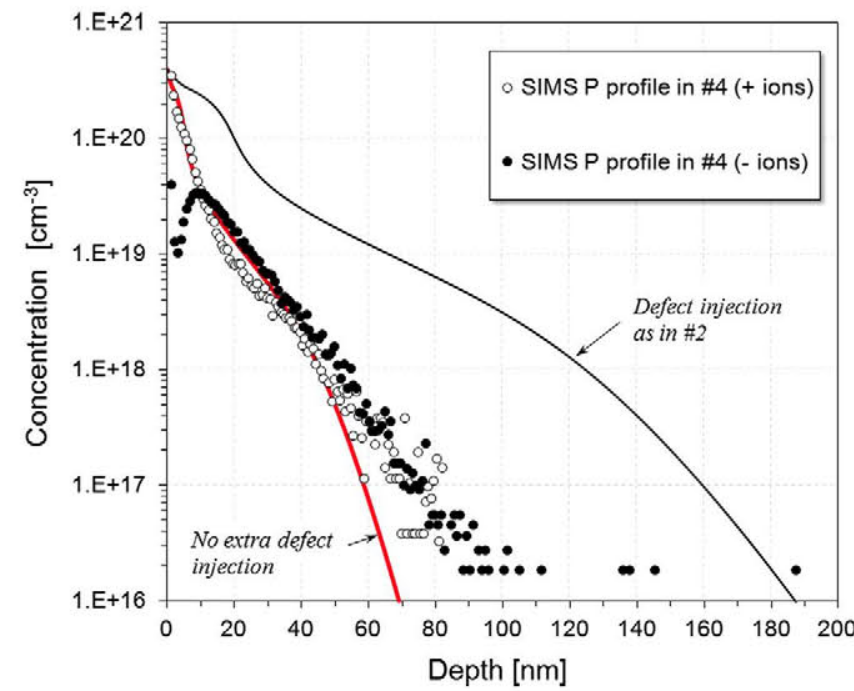

Fig. 4. Comparison of the phosphorus SIMS profiles measured (negative and positive ions) for sample \#4 and the profiles calculated with and without defect injection (solid lines). (For interpretation of the references to color in this figure, the reader is referred to the web version of this article.)

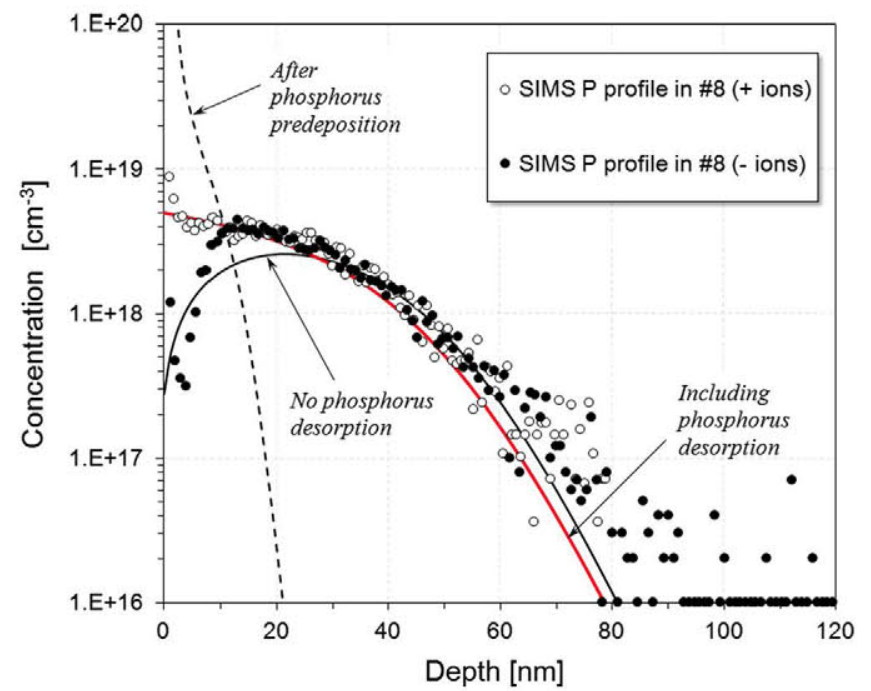

Fig. 5. Comparison of the phosphorus SIMS profiles measured (negative and positive ions) for sample \#8 and the profiles calculated with and without phosphorus desorption (solid lines). The calculated profile after P deposition has been also calculated for comparison (dashed line). (For interpretation of the references to color in this figure, the reader is referred to the web version of this article.)

thus without intensive surface displacement of silicon atoms) there is no enhanced diffusivity in the tail region.

The third group of samples studied in this work are those where a two-step thermal process (first a phosphorus predeposition followed by a drive-in) is carried out. The phosphorus redistribution during the drive-in step leads to much lower surface concentrations $\left(<10^{19} \mathrm{~cm}^{-3}\right.$ ), as confirmed by the SIMS profile of sample \#8 (reproduced in Fig. 5). This sample was exposed to our higher $\mathrm{PH}_{3}$ partial pressure conditions (32.1 mbar) for only $10 \mathrm{~min}$ at $830^{\circ} \mathrm{C}$, followed by a drive-in at $875^{\circ} \mathrm{C}$ in a $\mathrm{H}_{2}$ atmosphere for $90 \mathrm{~min}$. Therefore, according to the phenomenological explanation given, little surface degradation is expected due to the short exposure time to $\mathrm{PH}_{3}$ (a six-fold reduction as compared to samples \#1 to \#3) and thus no increase in the surface injection rates for vacancies and self-interstitials is expected. This assumption was indirectly confirmed by growing a new sample (\#9 in Table 2),
Table 4

Comparison of emitter depths from measurements and simulations.

\begin{tabular}{lll}
\hline Sample & $\begin{array}{l}\text { Emitter depth extrapolated from ECV } \\
(\mathrm{nm})\end{array}$ & $\begin{array}{l}\text { Emitter depth simulation } \\
(\mathrm{nm})\end{array}$ \\
\hline 1 & $260-274$ & $265-280$ \\
2 & $189-200$ & $194-201$ \\
3 & $173-183$ & $178-189$ \\
4 & $93-99$ & $71-80$ \\
5 & $130-141$ & $149-155$ \\
6 & $103-112$ & $122-126$ \\
7 & $80-87$ & $93-95$ \\
8 & $110-121$ & $86-90$ \\
\hline
\end{tabular}

which was only subjected to the predeposition step $\left(p_{\mathrm{PH} 3}=32.1 \mathrm{mbar}\right.$; for only $10 \mathrm{~min}$ at $830^{\circ} \mathrm{C}$ ) and then measured by AFM exhibiting a RMS roughness of $0.48 \mathrm{~nm}$, reasonably similar to those with little or no surface degradation (Table 2). The black solid line in Fig. 5 presents the simulated phosphorus profile for the two-step thermal treatment of sample \#8 using the nominal times and temperatures and a constant surface phosphorus concentration of $4 \times 10^{20} \mathrm{~cm}^{-3}$ during the predeposition step, in agreement with the values measured for the previous samples. In Fig. 5 we also included as a dashed line the simulation of the kink and tail profile obtained after the first predeposition step. Comparing the simulated (black solid line) and the measured profiles, the fit is fairly reasonable in the tail region. However, at the first nanometers, the simulation predicts lower phosphorus concentrations after such a prolonged dopant drive-in at $875^{\circ} \mathrm{C}$ for $90 \mathrm{~min}$. In fact, the simulation follows well the SIMS profile taken with negative ions which is known to produce an underestimation of the real surface concentration. The SIMS profile obtained with positive ions shows a nearly constant dopant concentration in the first $25 \mathrm{~nm}$. According to our simulations, this is only possible if a certain amount of $\mathrm{P}$ is made available at the surface during the drive-in step when no $\mathrm{PH}_{3}$ was injected. Repeating the simulations, a reasonably good fit is obtained considering a constant phosphorus concentration of $5 \times 10^{18} \mathrm{~cm}^{-3}$ during the second thermal step (red solid line in Fig. 5). We believe that this extra P supply is caused by atomic phosphorus which was deposited on the reactor walls and graphite parts during the predeposition step (at $830^{\circ} \mathrm{C}$ ) and is desorbed from the reactor walls and surfaces at the drive-in stage held at significantly higher temperatures $\left(875^{\circ} \mathrm{C}\right)$. We have some indirect evidence about the existence of this $\mathrm{P}$ background pressure since we routinely observe changes in surface dopant concentration of wafers after a long heat bake under pure $\mathrm{H}_{2}$ if the reactor has been coated with $P$ in previous runs. In summary, sample \#8 represents a case study were surface degradation has been controlled and no extra injection of point defects seems to be present. However, a well-known phenomenon-namely group V memory effect-to some extent inherent to any MOVPE reactor appears to influence the final $\mathrm{P}$ diffusion profile obtained.

As a final proof to test our diffusion model we calculated the junction depths for all samples in Table 1. Table 4 compares the junction depths estimated from SIMS and ECV profiles with the depths calculated from simulated $P$ profiles. As shown by this table, the agreement between both values is quite good, offering again an indirect proof of the robustness of the simulation model developed.

\section{Summary and conclusions}

Dual-junction solar cells formed by a GaAsP or GalnP top cell and a Si bottom cell seem to be excellent candidates for the integration of III-V materials on Si. This combination may provide a cost breakthrough for PV technology, unifying the low cost of Si 
and the efficiency potential of III-V MJSC. In this study, we have studied both by simulations and experimentally the formation of the emitter by the diffusion of phosphorus into the silicon substrate, during the MOVPE process. Wafers have been submitted to different treatments, where parameters such as temperature, time exposure or phosphine partial pressure, were varied. From SIMS and ECV P profiles we have confirmed that when large amounts of $\mathrm{PH}_{3}$ are present in the reactor during annealing the surface morphology of the samples degrades and the diffusion profiles obtained are much deeper. To be able to simulate such deep profiles, an extra injection of point defects, namely silicon vacancies and self-interstitials, at the surface has to be assumed. The origin of this phenomenon has been postulated to be the hydration of surface silicon atoms by $\mathrm{PH}_{3}$. On the contrary, when low partial pressures of $\mathrm{PH}_{3}$ are used (which do not cause surface morphology degradation due to the absence of a significant etch on the surface) the P diffusion profiles measured can be simulated without any extra addition of point defects. When the exposure to $\mathrm{PH}_{3}$ is short enough and is followed by an annealing under hydrogen (i.e. a drive-in), the degradation in morphology is minimum and the diffusion profiles associated to the redistribution of $\mathrm{P}$ can be calculated without considering extra injection of point defects but taking into consideration memory effects (i.e. $P$ desorption in the MOVPE reactor) that act as unintentional extra supply of $P$ atoms at the surface.

\section{Acknowledgments}

This work was supported by FP7 Program of the European Union through project NGCPV(283798) and by the Spanish Ministerio de Economía y Competitividad under project with reference TEC200911143.

\section{References}

[1] A. Martí, G.L. Araújo, Limiting efficiencies for photovoltaic energy conversion in multigap systems, Solar Energy Materials and Solar Cells 43 (1996) 203-222.

[2] C. Algora, Next Generation Photovoltaics: High Efficiency Through Full Spectrum Utilization, IOP Publishing, Bristol (United Kingdom), 2004 chapter 6.

[3] Y. Takano, T. Takagi, Y. Matsuo, S. Fuke, Surface evolution of GaP grown on Si substrates using metalorganic vapor phase epitaxy, Japanese Journal of Applied Physics 49 (2010) 035502-1-035502-5.

[4] I. Németh, B. Kunert, W. Stolz, K. Volz, Heteroepitaxy of GaP on Si: correlation of morphology, anti-phase-domain structure and MOVPE growth conditions, Journal of Crystal Growth 310 (2008) 1595-1601.

[5] V.K. Dixit, T. Ganguli, T.K. Sharma, R. Kumar, S. Porwal, V. Shukla, A. Ingale, P. Tiwari, A.K. Nath, Studies on MOVPE growth of GaP epitaxial layer on Si(001) substrate and effects of annealing, Journal of Crystal Growth 293 (2006) 5-13.

[6] T. Soga, T. Jimbo, M. Umeno, Initial stage of GaP/Si heteroepitaxial growth by metalorganic chemical vapor deposition, Journal of Crystal Growth 163 (1996) 165-170.

[7] T.J. Grassman, M.R. Brenner, M. Gonzalez, A.M. Carlin, R.R. Unocic, R.R. Dehoff, M.J. Mills, S.A. Ringel, Characterization of metamorphic GaAsP/Si materials and devices for photovoltaic applications, IEEE Transactions on Electron Devices 57 (2010) 3361-3369.
[8] T.J. Grassman, M.R. Brenner, A.M. Carlin, S. Rajagopalan, R. Unocic, R. Dehoff, M. Mills, H. Fraser, S.A. Ringel, Toward metamorphic multijunction GaAsP/Si photovoltaics grown on optimized GaP/Si virtual substrates using aniongraded GaAsP buffers, in: 34th IEEE Photovoltaic Specialists Conference (PVSC), Philadelphia, USA, 2009.

[9] J.F. Geisz, J.M. Olson, M.J. Romero, C.S. Jiang A.G. Norman, Lattice-mismatched GaAsP solar cells grown on silicon by OMVPE, in: 4th World Conference on Photovoltaic Energy Conversion, Hawaii, USA, 2006.

[10] J.F. Geisz, J.M. Olson, D.J. Friedman, Toward a monolithic lattice-matched III-V on silicon tandem solar cell, in: 19th European Photovoltaic Solar Energy Conference, Paris, France, 2004.

[11] K. Hayashi, T. Soga, H. Nishikawa, T. Jimbo, M. Umeno, MOCVD growth of GaAsP on Si for tandem solar cell application, in: 24th IEEE Photovoltaic Specialists Conference, First World Conference on Photovoltaic Energy Conversion, Hawaii, USA, 1994

[12] T. Roesener, H. Döscher, A. Beyer, S. Brückner, V. Klinger, A. Wekkeli, P. Kleinschmidt, C. Jurecka, J. Ohlmann, K. Volz, W. Stolz, T. Hannappel, A.W. Bett.F. Dimroth, MOVPE growth of Ill-V solar cells on silicon in $300 \mathrm{~mm}$ closed coupled showerhead reactor, in:25th European Photovoltaic Solar Energy Conference and Exhibition, Valencia, Spain, 2010.

[13] A. Bentzen, A. Holt, J.S. Christensen, B.G. Svensson, High concentration indiffusion of phosphorus in Si from a spray-on source, Journal of Applied Physics 99 (2006) 064502-1-064502-8.

[14] E. García-Tabarés, I. García, D. Martín, I. Rey-Stolle, Optimizing bottom subcells for III-V-on-Si multijunction solar cells, in: 37th IEEE Photovoltaic Specialists Conference (PVSC), Seattle, USA, 2011

[15] E.B. Kunert, I. Nemeth, S. Reinhard, K. Volz, W. Stolz, Si (001) surface preparation for the antiphase domain free heteroepitaxial growth of GaP on Si substrate, Thin Solid Films 517 (2008) 140-143.

[16] H. Döscher, S. Brückner, A. Dobrich, C. Höhn, P. Kleinschmidt, T. Hannappel, Surface preparation of $S i(100)$ by thermal oxide removal in a chemical vapor environment, Journal of Crystal Growth 315 (2011) 10-15.

[17] D.-S. Lin, T.-S. Ku, T.-J. Sheu, Thermal reactions of phosphine with Si(100): a combined photoemission and scanning-tunneling-microscopy study, Surface Science 424 (1999) 7-18.

[18] Y. Takano, T. Okamoto, T. Takagi, S. Fuke, Influence of $\mathrm{PH}_{3}$ preflow time on initial growth of $\mathrm{GaP}$ on $\mathrm{Si}$ substrates by metalorganic vapor phase epitaxy, IEICE Transactions on Electronics E92.C (12) (2009) 1443-1448.

[19] 1. Horcas, R. Fernández, J.M. Gómez-Rodríguez, J. Colchero, J. Gómez-Herrero, A.M. Baro, WSXM: a software for scanning probe microscopy and a tool for nanotechnology, Review of Scientific Instruments 78 (2007) 013705-1013705-8.

[20] E. Peiner, A. Schlachetzki, D. Krüger, Doping profile analysis in Si by electrochemical capacitance-voltage measurements, Journal of the Electrochemical Society 142 (2) (1995) 576-580, http://dx.doi.org/10.1149/1.2044101.

[21] A. Betzen, J.S. Christensen, B.G. Svensson, A. Hold, Understanding phosphorus emitter diffusion in silicon solar cell processing, in: Proceedings of the 21 th European Photovoltaic Solar Energy Conference, Dresden, Germany, 2006 pp. 1388-1391.

[22] S. Solmi, A. Parisini, R. Angelucci, A. Armigliato, Dopant and carrier concentration in Si in equilibrium with monoclinic SiP precipitates, Physical Review B 53 (1996) 7836-7841.

[23] H. Döscher, P. Kleinschmidt, T. Hannapel, Atomic structure of Si (100) substrates prepared in a chemical vapor environment, Applied Surface Science 257 (2010) 574-580

[24] P.M. Fahrey, P.B. Griffin, J.D. Plummer, Point defects and dopant diffusion in silicon, Modern Physics 61 (2) (1989) 289-384.

[25] /http://www.silvaco.com/products/process_simulation/athena_21.pdf)

[26] R.B. Fair, in: F.F.Y. Wang (Ed.), Impurity Doping Process in Silicon, North Holland, Amsterdam, 1981.

[27] H. Park, M. Law, Point defect based modeling of low dose silicon implant damage and oxidation effects on phosphorus and boron diffusion in silicon, Journal of Applied Physics 72 (1992) 3431-3439.

[28] E. García-Tabarés, I. García, I. Rey-Stolle, Optimization of the silicon subcell for III-V on silicon multijunction solar cells: key differences with conventional silicon technology, in: 8th International Conference on Concentrating Photovoltaic Systems, Toledo, Spain, 2012. 